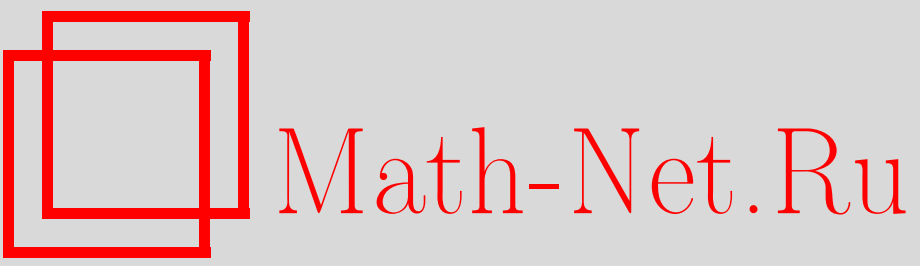

А. Кунду, Построение нового класса неоднородных квантовых интегрируемых моделей, ТМФ, 1999, том 118, номер 3, 423-433

DOI: https://doi.org/10.4213/tmf715

Использование Общероссийского математического портала Math-Net.Ru подразумевает, что вы прочитали и согласны с пользовательским соглашением

http://www . mathnet.ru/rus/agreement

Параметры загрузки:

IP : 18.209 .158 .208

26 апреля 2023 г., 15:58:05 
ТЕОРЕТИЧЕСКАЯ

И МАТЕМАТИЧЕСКАЯ

ФИЗИКА

Том 118, № 3

март, 1999

(C) 1999 г.

А. Кунду*

\title{
ПОСТРОЕНИЕ НОВОГО КЛАССА НЕОДНОРОДНЫХ КВАНТОВЫХ ИНТЕГРИРУЕМЫХ МОДЕЛЕЙ
}

\begin{abstract}
Построение неоднородных или примесных интегрируемых моделей обычно проводят или посредством сдвига спектрального параметра в операторе Лакса, или с использованием другого представления спиновой алгебры. В данной статье для такого построения предлагается менее прямой и более общий метод, в котором оператор Лакса содержит генераторы новой квадратичной алгебры, представляющей собой обобщение известной квантовой алгебры. При построении матрицы монодромии можно заменить любое число локальных операторов Лакса другими операторами, соответствуюшими различным реализациям исходной алгебры. Такая замена может привести к спиновым цепочкам с неспиновыми примесями, влияющими на изменение величины константы связи в примесном узле, а также к спиновым цепочкам, в которых примеси представлены бозонными операторами. В рамках данного подхода можно также построить интегрируемые неоднородные варианты обобщенной решеточной модели синус-Гордон, нелинейного уравнения Шредингера, модели Лиувилля, релятивистских и нерелятивистских цепочек Тоды и т. д.
\end{abstract}

\section{1. ВВЕДЕНИЕ}

Роль квантового метода обратной задачи рассеяния [1] в точно решаемых квантовых моделях трудно переоценить. Замечательная особенность данного метода состоит в том, что его можно применять к определенным моделям неоднородностей, не нарушая при этом интегрируемости исходной однородной модели. Обычно примеси вводятся через неоднородности в трансфер-матрице путем сдвига спектрального параметра [2], поскольку такой сдвиг не оказывает влияния на основное уравнение квантового метода обратной задачи рассеяния - квантовое уравнение Янга-Бакстера

$$
R_{12}(\lambda-\mu) L_{1 j}(\lambda) L_{2 j}(\mu)=L_{2 j}(\mu) L_{1 j}(\lambda) R_{12}(\lambda-\mu)
$$

обеспечивающее интегрируемость последнего. Такая процедура применялась некоторыми авторами к примесям в гейзенберговской спиновой цепочке [3] и к $t-j$-модели [4].

*Теоретическая группа Института Саха ядерной физики, 1/АF Бидхан Нагар, Калькутта 700 064, Индия. E-mail: anjan@tnp.saha.ernet.in 
Другой интересный подход к построению интегрируемой модели неоднородностей заключается в изменении представления исходной алгебры и, следовательно, оператора Лакса в примесных узлах. Такой подход впервые был использован в работе [5] для исследования влияния узла со спином $S$ в гейзенберговской цепочке спинов $1 / 2$, а также для построения альтернированных спиновых цепочек [6], $t$ - $j$-цепочек [7] и спиновых цепочек более высокого ранга [8]. Однако из-за использования представлений высокого ранга и появления членов взаимодействия большого числа тел многие из этих моделей становятся слишком сложными.

Для интегрируемых неоднородных моделей, включающих только операторы спина $1 / 2$, используют, как правило, первый метод, а именно, сдвиг спектрального параметра [9]. Это связано с тем, что выбор значения спина в исходной алгебре $s u(2)$ также ограничивает представление, а это не позволяет применять второй метод (т. е. использовать различные представления в различных узлах). Несмотря на то что рассматриваемый в данной статье подход основан на втором методе, он может быть применен и к случаю цепочек спинов $1 / 2$, а также ко всему классу неоднородных точно интегрируемых квантовых решеточных моделей. Успех подхода основан на более общей алгебраической сруктуре, лежащей в основе квантовых интегрируемых систем, а именно, на новой квантовой алгебре $U_{q}(K)$, допускающей различные типы представлений. Алгебра $U_{q}(K)$ является деформацией скорее квадратичной алгебры $K$, чем алгебры Ли, и соответствует обобшению хорошо известной квантовой алгебры $U_{q}(s u(2))$.

В данной статье предлагается новый класс конструкций для простейших спиновых цепочек спинов $1 / 2$, в котором в отличие от традиционного способа используются представления различных типов алгебр для различных узлов. В нашем случае это оказывается возможным вследствие того, что мы используем более общую структуру исходной алгебры, допускающей различные представления для одной и той же $R$-матришы. Таким образом, возможно рассматрение гейзенберговских цепочек спинов $1 / 2$ с неспиновыми примесями, что не приводит к появлению более чем двухчастичного взаимодействия, причем неоднородность играет существенную роль в изменении константы взаимодействия модели в примесных узлах. На этом пути удается описать локализованное антиферромагнитное взаимодействие в пределах общей ферромагнитной цепочки (или наоборот). Аналогичным образом можно рассмотреть наличие бозонной примеси в интегрируемой цепочке спинов $1 / 2$. Подобный подход к построению неоднородных моделей может быть обобщен, если полностью использовать свободу, предоставляемую исходной алгеброй. Такое обобшение может привести к новым, неоднородным вариантам решеточной модели синус-Гордон, нелинейного уравнения Шредингера, цепочек Тоды и т. д.

\section{2. ИСХОДНАЯ АЛГЕБРА В КВАНТОВЫХ ИНТЕГРИРУЕМЫХ СИСТЕМАХ}

Поскольку наше построение сильно зависит от исходной алгебры, необходимо сначала ввести саму эту алгебру, а потом уже рассматривать ее применения. Напомним, 
что квантовая алгебра Ли со свойствами некоммутативной алгебры Хопфа, привлекавшая значительное внимание исследователей в последнее время [10], в действительности обязана своим открытием квантовым интегрируемым системам $[11,12]$. Алгебра $U_{q}(s u(2))$ встречается уже в формулировке точно интегрируемой дискретной модели синус-Гордон $[13,14]$. Аналогично алгебра $q$-осцилляторов, хотя и была первоначально предложена как абстрактная реализация алгебры $U_{q}(s u(2))$ [15], оказалась связанной с нелинейным уравнением Шредингера (НУШ) с квантовыми производными [16], моделью Абловица-Ладика [17], а также с решеточным вариантом полевой модели Тоды [18]. Впоследствии было установлено, что целый ряд квантовых интегрируемых моделей удовлетворяет некоторой абстрактной алгебраической структуре [19].

Перечисленные выше факты побудили нас начать в квантовых интегрируемых системах поиск исходной алгебры более обшего вида для класса моделей, чьи $2 \times 2$-операторы Лакса имеют тригонометрическую или рациональную зависимость от спектральных параметров. Чтобы сохранялась квантовая интегрируемость, эта алгебра должна следовать из уравнения Янга-Бакстера, а также обладать требуемыми свойствами алгебры Хопфа. Более того, такая алгебра должна не только обобшать известную квантовую алгебру $U_{q}(s u(2))$, но и порождать другие алгебры, такие как алгебру $q$-осцилляторов, $s u(2)$-алгебру, гейзенберговскую алгебру и т. д., существующие, как известно, в интегрируемых моделях.

Чтобы удовлетворить перечисленным выше требованиям, возьмем операторы Лакса в виде матриц с операторнозначными матричными элементами

$$
L_{t}(\xi)=\left(\begin{array}{cc}
\xi c_{1}^{+} e^{i \alpha S^{3}}+\xi^{-1} c_{1}^{-} e^{-i \alpha S^{3}} & 2 \sin \left(\alpha S^{-}\right) \\
2 \sin \left(\alpha S^{+}\right) & \xi c_{2}^{+} e^{-i \alpha S^{3}}+\xi^{-1} c_{2}^{-} e^{i \alpha S^{3}}
\end{array}\right), \quad \xi=e^{i \lambda}
$$

Эти операторы представляют собой абстрактную интегрируемую систему, если соответствуюшую $R$-матрицу определить как известное решение с ненулевыми элементами

$$
R_{11}^{11}=R_{22}^{22}=a(\lambda), \quad R_{12}^{12}=R_{21}^{21}=b(\lambda), \quad R_{21}^{12}=R_{12}^{21}=c,
$$

выраженными через тригонометрические функции спектральных параметров

$$
a(\lambda)=\sin (\lambda+\alpha), \quad b(\lambda)=\sin \lambda, \quad c=\sin \alpha
$$

а также если удовлетворяется уравнение Янга-Бакстера (1.1). В свою очередь, условие интегрируемости (1.1) определяет алгебру

$$
\left[S^{3}, S^{ \pm}\right]= \pm S^{ \pm}, \quad\left[S^{+}, S^{-}\right]=\frac{1}{2}\left(M^{+}\left[2 S^{3}\right]_{q}-i \frac{M^{-}}{\sin \alpha} \cos \left(2 \alpha S^{3}\right)\right)
$$

где $[x]_{q}=\left(q^{x}-q^{-x}\right) /\left(q-q^{-1}\right)=(\sin (\alpha x)) /(\sin \alpha), q=e^{i \alpha}$, а элементы $M^{ \pm}$связаны с центральными элементами $\left\{c_{a}^{ \pm}\right\}$алгебры соотношением $M^{ \pm}=c_{1}^{+} c_{2}^{-} \pm c_{1}^{-} c_{2}^{+}$. 
Заметим, что алгебра (2.4) представляет собой обобщение алгебры $U_{q}(s u(2))$ и, в отличие от случая алгебр Ли или их деформаций, в алгебраических соотношениях появляются мультипликативные центральные элементы $M^{ \pm}$, принимаюшие произвольные значения. Обозначим новую алгебру, представляюшую собой $q$-деформацию квадратичной алгебры $K$, как $U_{q}(K)$. Впервые понятие квадратичной алгебры было введено в работе [20].

Для алгебры (2.4) можно построить основные элементы, задающие структуру алгебры Хопфа, такие как копроизведение $\Delta(x): A \rightarrow A \otimes A$, определяемое соотношениями

$$
\begin{aligned}
\Delta\left(S^{+}\right) & =c_{1}^{+} e^{i \alpha S^{3}} \otimes S^{+}+S^{+} \otimes c_{2}^{+} e^{-i \alpha S^{3}}, \\
\Delta\left(S^{-}\right) & =c_{2}^{-} e^{i \alpha S^{3}} \otimes S^{-}+S^{-} \otimes c_{1}^{-} e^{-i \alpha S^{3}}, \\
\Delta\left(S^{3}\right) & =I \otimes S^{3}+S^{3} \otimes I, \\
\Delta\left(c_{i}^{ \pm}\right) & =c_{i}^{ \pm} \otimes c_{i}^{ \pm}
\end{aligned}
$$

антипод $S: A \rightarrow A$ (или “взятие обратного"),

$$
\begin{aligned}
& S\left(S^{-}\right)=-\left(c_{1}^{+}\right)^{-1} e^{-i \alpha S^{3}} S^{-} e^{i \alpha S^{3}}\left(c_{2}^{+}\right)^{-1}, \\
& S\left(S^{+}\right)=-\left(c_{2}^{-}\right)^{-1} e^{-i \alpha S^{3}} S^{+} e^{i \alpha S^{3}}\left(c_{1}^{-}\right)^{-1}, \\
& S\left(c_{i}^{ \pm}\right)=\left(c_{i}^{ \pm}\right)^{-1}, \quad S\left(e^{ \pm i \alpha S^{3}}\right)=e^{\mp i \alpha S^{3}},
\end{aligned}
$$

а также коединицу $\epsilon: A \rightarrow k$, отображаюшую алгебру в числовые тождества: $\epsilon\left(c_{i}^{ \pm}\right)=1$, $\epsilon\left(e^{ \pm i \alpha S^{3}}\right)=1$ и $\epsilon\left(S^{ \pm}\right)=0$. Аналогично можно определить операцию умножения $M: A \otimes A \rightarrow A$ и единицу $\eta: k \rightarrow A$. Совокупность этих элементов преврашает алгебру (2.4) в алгебру Хопфа. Заметим, что структура алгебры Хопфа наряду с той ролью, которую она играет по отношению к симметриям, является также ключевым свойством при выводе того же самого соотношения Янга-Бакстера (1.1) для глобального объекта $[9]$

$$
T(\lambda)=\prod_{i=1}^{N} L_{i}(\lambda)
$$

а именно

$$
R_{12}(\lambda-\mu) T_{1}(\lambda) T_{2}(\mu)=T_{2}(\mu) T_{1}(\lambda) R_{12}(\lambda-\mu) .
$$

Отсюда получаем для генераторов сохраняюшихся величин соотношение

$$
t(\lambda)=\operatorname{tr} T(\lambda)=\sum_{n}^{\infty} C_{n} \lambda^{n}
$$

которое удовлетворяет условию квантовой интегрируемости $[t(\lambda), t(\mu)]=0$.

Теперь покажем, что, взяв алгебру (2.4) в качестве основной алгебры в тригонометрическом классе интегрируемых моделей, можно получить все другие известные алгебры, ассоциированные с этим классом моделей. Для этого нужно правильным образом 
выбрать центральный элемент. Например, выбрав центральные элементы симметричными, т.е.

$$
c_{a}^{ \pm}= \pm q^{ \pm \frac{1}{2}}, \quad a=1,2,
$$

что дает $M^{-}=0, M^{+}=-2$, и переопределив оператор $S^{ \pm}$(умножив его на $i$ ), получаем известную квантовую алгебру $U_{q}(s u(2))[11]$

$$
\left[S^{3}, S^{ \pm}\right]= \pm S^{ \pm}, \quad\left[S^{+}, S^{-}\right]=\left[2 S^{3}\right]_{q},
$$

лежашую в основе модели синус-Гордон, решеточной модели синус-Гордон, гейзенберговской спиновой $X X Z$-цепочки и т. д. [12].

Несколько асимметричный выбор центральных элементов

$$
c_{1}^{+}=c_{2}^{+}=1, \quad c_{1}^{-}=-i q, \quad c_{2}^{-}=\frac{i}{q},
$$

дающий $M^{+}=2 \sin \alpha, M^{-}=2 i \cos \alpha$, вместе с отображением

$$
S^{+}=-\kappa A, \quad S^{-}=\kappa A^{\dagger}, \quad S^{3}=-N, \quad \kappa=-i(\operatorname{ctg} \alpha)^{\frac{1}{2}},
$$

сводит $U_{q}(K)$ к известной алгебре $q$-осцилляторов $[15,21]$

$$
[A, N]=A, \quad\left[A^{\dagger}, N\right]=-A^{\dagger}, \quad\left[A, A^{\dagger}\right]=\frac{\cos (\alpha(2 N+1))}{\cos \alpha},
$$

свойственной таким квантовым интегрируемым моделям, как производное НУШ, модель Абловица-Ладика и т. д. [19].

Другая важная трехмерная алгебра Ли может быть получена из алгебры $U_{q}(K)$, если положить

$$
c_{1}^{+}=c_{2}^{-}=1, \quad c_{1}^{-}=c_{2}^{+}=0
$$

и выбрать $M^{+}=M^{-}=1$. При этом возникает әкспоненииально деформированная алгебра Ли

$$
\left[S^{+}, S^{-}\right]=\frac{e^{2 i \alpha S^{3}}}{2 i \sin \alpha},
$$

соответствуюшая квантовой модели Лиувилля [22]. С другой стороны, если выбрать $M^{ \pm}=0$, получается неабелева алгебра

$$
\left[S^{3}, S^{ \pm}\right]= \pm S^{ \pm}, \quad\left[S^{+}, S^{-}\right]=0
$$

связанная с квантовой интегрируемой релятивистской цепочкой Тоды или с цепочкой Тоды с дискретным временем [23].

Рассмотрим теперь недеформированную алгебру $K$, получаемую из алгебры $(2.4)$ в пределе $q \rightarrow 1$. При этом для конечности предела требуется, чтобы центральные элементы зависели от параметра $q$. Согласованная процедура приводит к алгебре

$$
\left[s^{+}, s^{-}\right]=2 m^{+} s^{3}+m^{-}, \quad\left[s^{3}, s^{ \pm}\right]= \pm s^{ \pm},
$$


где $m^{+}=c_{1}^{0} c_{2}^{0}, m^{-}=c_{1}^{1} c_{2}^{0}+c_{1}^{0} c_{2}^{1}$, а операторы $c_{i}^{a}$ являются новыми центральными элементами. Заметим, что эта алгебра снова не есть алгебра Ли. Она является квадратичной алгеброй с произвольными мультипликативными элементами $m^{ \pm}$. Соответствуюшие копроизведения можно получить из соотношений (2.5), устремив $\alpha \rightarrow 0$,

$$
\begin{aligned}
\Delta\left(s^{+}\right) & =c_{1}^{0} \otimes s^{+}+s^{+} \otimes c_{2}^{0}, & \Delta\left(s^{-}\right) & =c_{2}^{0} \otimes s^{-}+s^{-} \otimes c_{1}^{0}, \\
\Delta\left(c_{i}^{0}\right) & =c_{i}^{0} \otimes c_{i}^{0}, & \Delta\left(c_{i}^{1}\right) & =c_{i}^{0} \otimes c_{i}^{1}+c_{i}^{1} \otimes c_{i}^{0},
\end{aligned}
$$

что дает $\Delta\left(m^{+}\right)=m^{+} \otimes m^{+}, \Delta\left(m^{-}\right)=m^{+} \otimes m^{-}+m^{-} \otimes m^{+}$. Необычное свойство соотношений (2.17) состоит в том, что хотя определяемая ими алгебра являетя недеформированной, по своей природе она некокоммутативна. Интегрируемые системы, связанные с этой алгеброй, принадлежат к другому, например рациональному, классу, поскольку квантовая $R$-матрица (2.2) при $q \rightarrow 1$ приобретает рациональный вид, где

$$
a(\lambda)=\lambda+\alpha, \quad b(\lambda)=\lambda, \quad c=\alpha,
$$

а оператор Лакса (2.1) приводится к виду

$$
L_{r}(\lambda)=\left(\begin{array}{cc}
c_{1}^{0}\left(\lambda+s^{3}\right)+c_{1}^{1} & s^{-} \\
s^{+} & c_{2}^{0}\left(\lambda-s^{3}\right)-c_{2}^{1}
\end{array}\right) .
$$

Как можно заметить, алгебра (2.16) представляет собой обобщение стандартной алгебры $s u(2)$ и сводится к ней, если выбрать $m^{+}=1, m^{-}=0$,

$$
\left[s^{3}, s^{ \pm}\right]= \pm s^{ \pm}, \quad\left[s^{+}, s^{-}\right]=2 s^{3}
$$

что связано с $X X X$-цепочками спинов $1 / 2$.

С другой стороны, выбор $m^{+}=0, m^{-}=1$ сводит алгебру (2.16) к бозонной алгебре

$$
[a, N]=a, \quad\left[a^{\dagger}, N\right]=-a^{\dagger}, \quad\left[a, a^{\dagger}\right]=1,
$$

которую можно связать с решеточной моделью НУШ [24]. Выбирая $m^{ \pm}=0$, мы получаем простую алгебру

$$
\left[s^{3}, s^{ \pm}\right]= \pm s^{ \pm}, \quad\left[s^{+}, s^{-}\right]=0
$$

лежашую в основе модели интегрируемых цепочек Тоды [19]. 


\section{3. ПОСТРОЕНИЕ НОВЫХ ГЕЙЗЕНБЕРГОВСКИХ ЦЕПОЧЕК С ПРИМЕСЯМИ}

Теперь, используя для различных примесных узлов различные реализации исходной квадратичной алгебры (2.4) или (2.16), зависяшие от класса моделей (например, тригонометрических или рациональных), мы готовы к построению неоднородных или примесных моделей.

Особый интерес представляют гейзенберговские цепочки спинов $1 / 2$ с примесями, поскольку наша исходная алгебра является более обшим объектом, чем стандартная спиновая алгебра (2.20), что дает нам больше возможностей для проведения подобных построений. В отличие от обычного подхода мы можем построить цепочки спинов $1 / 2$ с неспиновыми примесями, а также с примесями, представленными бозонными операторами (2.21). Рассмотрим сначала простейший пример спиновой $X X Z$-цепочки и влияние неспиновой примеси в $m$-м узле. Заметим, что случай $\vec{S}=0$ нетривиальным образом включается в $(2.1)$, если там выбрать $c_{1}^{ \pm}= \pm e^{ \pm i c_{m}^{1}}$ и $c_{2}^{ \pm}=\mp e^{ \pm i c_{m}^{1}}$. Соответственно аналогично тому, как это было сделано в (2.7), в цепочке $X X Z$-операторов Лакса (полученных из матриц (2.1) с помошью (2.8) и спиновых операторов, взятых в виде матриц Паули, удовлетворяюших соотношениям (2.20)) и в матрище монодромии $T(\lambda)$ мы заменили оператор $L$ в $m$-м узле решетки на $L_{a m}=\sin \left(\lambda+c_{m}^{1}\right) \sigma_{a}^{3}$.

Операторы Лакса, сохраняющие исходную алгебру (2.4) и имеющие ту же тригонометрическую $R$-матрицу, определяют согласованную интегрируемую систему. Гамильтониан такой интегрируемой спиновой цепочки, содержащей примесь или дефект, может быть получен обычным способом как $H=C_{1}=t^{\prime}(0) t^{-1}(0)$, откуда следует, что

$$
H=-\left(\sum_{j \neq m, m-1} h_{j j+1}^{\mathrm{Xxz}}-\left(\sigma_{m-1}^{+} \sigma_{m+1}^{-}+\sigma_{m-1}^{-} \sigma_{m+1}^{+}\right)+\cos \alpha \sigma_{m-1}^{3} \sigma_{m+1}^{3}\right) .
$$

Здесь $X X Z$-гамильтониан записан в известном виде

$$
h_{j j+1}^{\mathrm{XXz}}=\sigma_{j}^{+} \sigma_{j+1}^{-}+\sigma_{j}^{-} \sigma_{j+1}^{+}+\cos \alpha \sigma_{j}^{3} \sigma_{j+1}^{3}
$$

Интересно заметить, что влияние неспиновой примеси состоит лиш в изменении величины связи в примесном узле, тогда как для $m$-го узла оно приводит к добавлению несушественного постоянного члена к гамильтониану. Аналогично можно получить примесную спиновую $X X X$-цепочку, если взять предельный случай $\alpha=0$, т. е. положить $\cos \alpha=1$ в уравнении (3.1).

Мы показали, что и спиновая алгебра (2.20) и бозонная алгебра (2.21) могут быть получены как различные реализации исходной алгебры (2.16). Можно, таким образом, не нарушая интегрируемости системы, в любом узле $X X X$-цепочки спинов $1 / 2$ (например, при $j=m$ ) заменить оператор Лакса $L_{j}^{\mathrm{XXX}}$ на операторы $L_{m}^{b}$ (включающие бозонные операторы); тогда ассоциированная квантовая $R$-матрица будет иметь рациональный 
вид (2.18). В частности, выбрав в уравнении (2.16) $c_{1}^{0}=c_{1}^{1}=c_{1}^{2}=1$ и $c_{2}^{0}=0$, мы получим бозонную алгебру. Поэтому при тех же значениях центральных элементов и при отображении $s^{+}=a, s^{-}=a^{\dagger}, s^{3}=-N$ оператор Лакса (2.19) принимает вид

$$
L_{m}^{b}(\lambda)=\left(\begin{array}{cc}
\left(\lambda-N_{m}\right)+1 & a_{m}^{\dagger} \\
a_{m} & -1
\end{array}\right)
$$

Этот оператор мы и помешаем в примесный узел. Следовательно, как и раньше, гамильтониан бозонной примеси в изотропной спиновой цепочке будет иметь вид

$$
\begin{aligned}
& H=-\left(\sum_{j \neq m, m-1} L^{\mathrm{XXX}_{(0)}}\right)_{j j+1}^{\prime}\left(L^{\mathrm{XXX}}(0)\right)_{j j+1}^{-1}+L^{b}(0)_{m m+1}^{\prime}\left(L^{b}(0)\right)_{m m+1}^{-1}+ \\
& \left.+L^{b}(0)_{m m-1}\left(L^{\mathrm{XXx}}(0)_{m+1 m-1}^{\prime} L^{\mathrm{XXx}}(0)_{m+1 m-1}^{-1}\right) L^{b}(0)_{m m-1}^{-1}\right),
\end{aligned}
$$

что можно также записать в более явной форме

$$
H=-\sum_{j \neq m, m-1} h_{j j+1}^{\mathrm{XXX}}+h_{m m+1}+h_{m-1 m m+1}
$$

Здесь $h_{j j+1}^{\mathrm{XXX}}$ соответствует известной изотропной спиновой цепочке, а

$$
\begin{aligned}
h_{m m+1}= & \frac{1}{2} \sigma_{m+1}^{3}-a_{m}^{\dagger} \sigma_{m+1}^{+}, \\
h_{m-1 m m+1}= & \sigma_{m-1}^{+} \sigma_{m+1}^{-}\left(1-N_{m}\right)^{2}-P_{m-1}^{+} a_{m} \sigma_{m+1}^{-}+\sigma_{m-1}^{-} \sigma_{m+1}^{+}+ \\
& +P_{m-1}^{-} a_{m}^{\dagger} \sigma_{m+1}^{+}+P_{m-1}^{-} a_{m}\left(1-N_{m}\right) \sigma_{m+1}^{-}-P_{m-1}^{+} a_{m}^{\dagger} \sigma_{m+1}^{+}- \\
& -\sigma_{m-1}^{+}\left(a_{m}^{\dagger}\right)^{2} \sigma_{m+1}^{+}-\sigma_{m-1}^{-} a_{m}^{2} \sigma_{m+1}^{-}
\end{aligned}
$$

где $P^{ \pm}=(1 / 2)\left(1 \pm \sigma^{3}\right)$

\section{4. НОВЫЕ НЕОДНОРОДНЫЕ МОДЕЛИ, ПОСТРОЕННЫЕ НА ОСНОВЕ ВЫБРАННОЙ АЛГЕБРЫ}

Мы показали, что алгебры $U_{q}(K)$ и $K$ могут служить алгебрами общего вида для квантовых интегрируемых систем. Аналогичным образом можно построить обобщения решеточной модели синус-Гордон, решеточного НУШ, цепочки Тоды и т. д., а также рассмотреть их неоднородные варианты.

Отметим, что интересная реализация квадратичной алгебры (2.4)в канонических переменных $u$ и $p$, удовлетворяюших соотношению $[u, p]=i$, которая использует полную свободу, предоставляемую алгеброй, может быть получена в виде

$$
S^{3}=u, \quad S^{+}=e^{-i p} g(u), \quad S^{-}=\left(S^{+}\right)^{\dagger},
$$


где

$$
g^{2}(u)=\frac{1}{2}\left(\frac{\kappa}{2 \sin ^{2} \alpha}+[s-u]_{q}\left(M^{+}[u+s+1]_{q}-\frac{i M^{-}}{\sin \alpha} \cos \alpha(u+s+1)\right)\right),
$$

$\kappa$ - произвольная постоянная. Вместе с оператором Лакса $(2.1)$ и квантовой $R$-матрицей (2.3) соотношения (4.1), (4.2) определяют новую обобшенную дискретную модель синус-Гордон, аналогичную модели, основанной на алгебре (2.4). Взяв центральные элементы равными $M^{+}=m^{2}$ и $M^{-}=0$, мы приходим к известной решеточной модели синус-Гордон [13]. С другой стороны, если в операторе Лакса (2.1) заменить все $c_{a}^{ \pm}$ на $c_{a j}^{ \pm}$, а в уравнении $(4.2)$ - все $M^{ \pm}$на $M_{j}^{ \pm}$(т. е. для каждого узла решетки выбрать различные центральные элементы), то получится новый тип неоднородной интегрируемой модели, соответствующей той же самой квантовой $R$-матрице (2.3). Даже в случае известной решеточной модели синус-Гордон вместо соотношений (2.8) можно использовать зависящие от узла величины $c_{a}^{ \pm}=m_{j} e^{i \epsilon_{a} \alpha \theta_{j}}, \epsilon_{1}=1$ и $\epsilon_{2}=-1$, что приводит к $M_{j}^{-}=0$ и $M_{j}^{+}=m_{j}^{2}$. В непрерывном пределе из такой неоднородной модели получается интегрируемая полевая модель синус-Гордон с переменной массой $m(x)$ во внешнем калибровочном поле $\theta(x)$.

Аналогичным образом можно использовать полную свободу, предоставляемую недеформированной алгеброй $K(2.16)$, для того чтобы получить реализацию квадратичной алгебры в виде обобшенного преобразования Холстейна-Примакова

$$
\begin{gathered}
s^{3}=s-N, \quad s^{+}=f(N) \psi, \quad s^{-}=\left(s^{+}\right)^{\dagger}, \\
f^{2}(N)=m^{-}+m^{+}(2 s-N), \quad N=\psi^{\dagger} \psi,
\end{gathered}
$$

где $\left[\psi, \psi^{\dagger}\right]=1$. Это преобразование является квантовой интегрируемой обобшенной решеточной моделью НУШ, получаемой при помоши оператора Лакса (2.19) и $R$-матрицы (2.18). Для $m^{-}=0$ и $m^{+}=1$ мы снова получаем стандартную решеточную модель НУШ [13]. Теперь можно построить неоднородную решеточную модель НУШ, подставив в оператор (2.19) зависяшие от узла решетки центральные элементы и заменив $m^{ \pm} \rightarrow m_{j}^{ \pm}$в обобшенном преобразовании Холстейна-Примакова (4.3). Если рассматривать данную модель как полевую, то с ее помощью можно было бы получить уравнения типа НУШ с переменными коэффициентами или цилиндрического НУШ [25]. Следует также заметить, что бозонную реализацию операторов Лакса общего вида (2.1) и (2.19) можно также найти в работе [14].

Аналогичным образом несложно получить неоднородные варианты и других интегрируемых моделей, таких как модель Лиувилля, релятивистские и нерелятивистские цепочки Тоды и т. д. Например, нерелятивистская цепочка Тоды может быть представлена как реализация алгебры (2.16) с помошью отображения

$$
s_{j}^{3}=p_{j}, \quad s_{j}^{+}=e^{-u_{j}}, \quad s_{j}^{-}=e^{u_{j}}, \quad c_{2}^{a}=0, \quad c_{1}^{a}=c_{j}^{a} .
$$

Соответствуюший гамильтониан имеет вид

$$
H=\sum_{j}\left(p_{j}+c_{j}^{1}\right)^{2}+\frac{1}{c_{j}^{0} c_{j+1}^{0}} e^{u_{j}-u_{j+1}},
$$

причем неоднородность определяется элементами $c_{j}^{a}$. 


\section{5. ЗАКЛЮЧИТЕЛЬНЫЕ ЗАМЕЧАНИЯ}

Таким образом, мы показали, что с помощью новых квадратичных алгебр в качестве исходной алгебраической структуры в квантовых интегрируемых моделях можно построить новьй класс неоднородных или примесных моделей. Используя различные представления этих алгебр, можно систематически строить как известные, так и новые квантовые интегрируемые системы.

Более того, поскольку алгебраический бете-анзац [1], являющийся точным методом решения задачи на собственные значения, главным образом зависит от элементов $R$-матрицы, все интегрируемые модели, включая примесные, можно решить с помощью почти универсального уравнения, основанного на модели обшего вида. Таким образом, допуская применимость алгебраического анзаца Бете (т. е. сушествование псевдовакуума для модели обшего вида (2.1) с $R$-матрищей (2.2)), мы можем получить следуюшее уравнение на собственные значения:

$$
\Lambda_{m}(\lambda)=\alpha(\lambda) \prod_{j=1}^{m} f\left(\lambda_{j}-\lambda\right)+\beta(\lambda) \prod_{j=1}^{m} f\left(\lambda-\lambda_{j}\right), \quad \text { где } \quad f(\lambda)=\frac{a(\lambda)}{b(\lambda)} .
$$

Здесь коэффициенты $\alpha(\lambda)$ и $\beta(\lambda)$ - псевдовакуумные собственные значения диагональных элементов оператора $T(\lambda)$, т. е. $T_{11}(\lambda)|0\rangle=\alpha(\lambda)|0\rangle$ и $T_{22}(\lambda)|0\rangle=\beta(\lambda)|0\rangle$; следовательно, это единственные элементы, зависящие от конкретной формы оператора Лакса. Зная, как выбрать центральные элементы в операторе Лакса, а также различные реализации этого оператора в различных моделях или в одной и той же модели с примесью, мы можем легко в каждом из случаев определить коэффициенты $\alpha(\lambda)$ и $\beta(\lambda)$. С другой стороны, значение выражения (5.6) определяется в основном произведениями

$$
\prod_{j=1}^{m} f\left(\lambda-\lambda_{j}\right)
$$

зависящими только от отношения $(a(\lambda)) /(b(\lambda))$ элементов $R$-матрицы $(2.2)$, которое является универсальным для всех моделей, принадлежащих к данному классу.

Обобшению предложенного подхода на случай алгебр более высоких рангов и других типов алгебр следует посвятить отдельную работу.

\section{Список литературы}

[1] L. D. Faddeev. Sov. Sci. Rev. C. 1980. V. 1. P. 107; J. H. Lowenstein. In: Les Houches Lect. Notes, 1984. P. 565.

[2] H. J. de Vega. Hidden gauge invariances in integrable theories. Preprint CERN-TH.4625. Geneva: CERN, 1986.

[3] P. Schmitteckert, P. Schwab, U. Eckern. Europhys. Lett. 1995. V. 30. P. 543; H. P. Eckle, A. Punnoose, R. Römer. Europhys. Lett. 1997. V. 39. P. 293.

[4] P. A. Bares. Exact result for a one dimensional $t-j$ model with impurities. cond-mat/9412011 (unpublished).

[5] N. Andrei, H. Johannesson. Phys. Lett. A. 1984. V. 100. P. 108. 
[6] H. J. de Vega, F. Woynarovich. J. Phys. A. 1992. V. 25. P. 4499.

[7] G. Bedürftig, F. Essler, H. Frahm. Nucl. Phys. B. 1997. V. 489. P. 697.

[8] J. Abad, M. Rios. Phys. Rev. B. 1996. V. 53. P. 14000.

[9] H. J. de Vega. Int. J. Mod. Phys. A. 1989. V. 4. P. 2371.

[10] V. Chari, A. Pressley. A guide to Quantum Groups. Cambridge: Cambridge Univ. Press, 1994.

[11] V. G. Drinfeld. Quantum groups. In: Proc. Int. Congr. Math. Berkeley, CA, 1986. Ed. A. M. Gleason. Providence: AMS, 1987. V. 1. P. 798.

[12] L. D. Faddeev. Int. J. Mod. Phys. A. 1995. V. 10. P. 1845.

[13] A. G. Izergin, V. E. Korepin. Nucl. Phys. B. 1982. V. 205 [FS 5]. P. 401.

[14] B. O. Тарасов. ТМФ. 1985. Т. 63. C. 175.

[15] A. J. Macfarlane. J. Phys. A. 1989. V. 22. P. 4581; L. C. Biederharn. J. Phys. A. 1989. V. 22. P. L873.

[16] A. Kundu, B. Basumallick. J. Math. Phys. 1993. V. 34. P. 1252.

[17] P. Kulish, E. K. Sklyanin. Quantum spectral transform method. Recent developments. In: Lect. Notes in Phys. V. 151. Integrable Quantum Field Theory: Proc. of the Symposium Held at Tvärminne, Finland, 23-27 March, 1981. Eds J. Hietarinta and C. Montonen. Berlin: Springer, 1982. P. 61.

[18] O. Babelon. Nucl. Phys. B [FS 10]. 1984. V. 230. P. 241.

[19] A. Kundu, B. Basumallick. Mod. Phys. Lett. A. 1992. V. 7. P. 61.

[20] Е. К. Склянин. Функц. анализ и его прилож. 1982. Т. 16. № 4. С. 27.

[21] Y. J. Ng. J. Phys. A. 1990. V. 23. P. 1023.

[22] L. D. Faddeev, L. A. Takhtadzhyan (Takhtajan). Liouville model on the lattice. In: Lect. Notes in Phys. V. 246. Field Theory, Quantum Gravity and Strings: Proc. of a Seminar Series Held at DAPHE, Observatoire de Meudon, and LPTHE, Université Pierre et Marie Curie, Paris, Between October, 1984 and October, 1985. Eds H. J. de Vega and N. Sanchez. Berlin: Springer, 1986. P. 166.

[23] A. Kundu. Phys. Lett. A. 1994. V. 190. P. 73.

[24] A. Kundu, O. Ragnisco. J. Phys. A. 1994. V. 27. P. 6335.

[25] R. Radha, M. Lakshmanan. J. Phys. A. 1995. V. 28. P. 6977. 